\title{
Vitamin D: a brief overview of its importance and role in inflammatory bowel disease
}

\author{
Kiran Mudambi, Dorsey Bass \\ Division of Pediatric Gastroenterology, Hepatology, and Nutrition, Stanford University School of Medicine, Stanford, USA \\ Contributions: (I) Conception and design: All authors; (II) Administrative support: None; (III) Provision of study materials or patients: None; (IV) \\ Collection and assembly of data: None; (V) Data analysis and interpretation: None; (VI) Manuscript writing: All authors; (VII) Final approval of \\ manuscript: All authors. \\ Correspondence to: Kiran Mudambi. Division of Pediatric Gastroenterology, Hepatology, and Nutrition, Stanford University School of Medicine, \\ Stanford, USA. Email: kiranm@stanford.edu.
}

\begin{abstract}
Vitamin D has traditionally been known for its regulation of bone metabolism and homeostasis, but emerging evidence suggests that it also has a broad function in immune regulation, and inflammatory bowel disease (IBD). The etiology of IBD is thought to be multifactorial but stems in part due to the deregulation of the immune response to environmental factors in the setting of a pre-existing genetic disposition. Vitamin D, based on its mechanistic role at the cellular level in T-cell trafficking, had been postulated to have a direct effect on the immune system, This alludes to the fact that vitamin D may have the ability to not only potentiate the IBD phenotype, but also in doing so, its supplementation may serve a therapeutic role in amelioration of the diseased state. We review in this article the current literature as it pertains to the basic mechanism of Vitamin D, its role in the pathogenicity of IBD, how it regulates our immune system, interpretation and accuracy of obtaining levels, and the role there may be in supplementation in IBD.
\end{abstract}

Keywords: Vitamin D; inflammatory bowel disease (IBD)

Received: 26 April 2018; Accepted: 03 May 2018; Published: 29 May 2018.

doi: $10.21037 / \operatorname{tgh} .2018 .05 .03$

View this article at: http://dx.doi.org/10.21037/tgh.2018.05.03

\section{Introduction}

Vitamin D has traditionally been known for its regulation of bone metabolism and homeostasis, but emerging evidence suggests that it also has a broad function in immune regulation, as noted by the fact that vitamin D deficiency has been associated with the pathogenesis of autoimmune and rheumatoid disorders, and more recently inflammatory bowel disease (IBD) (1). The etiology of IBD is thought to be multifactorial but stems in part due to the deregulation of the immune response to environmental factors in the setting of a pre-existing genetic disposition. Vitamin D, based on its mechanistic role at the cellular level in T-cell trafficking, had been postulated to have a direct effect on the immune system, which we now know is scientifically true. This alludes to the fact that vitamin D may have the ability to not only potentiate the IBD phenotype, but also in doing so, its supplementation may serve a therapeutic role in amelioration of the diseased state.

Vitamin D comprises a group of fat-soluble hormones, which include ergocalciferol (vitamin D2) and cholecalciferol (vitamin D3) (1). Ultraviolet B radiation converts cutaneous 7-dehydrocholesterol, a zoosterol precursor found in our skin, to cholecalciferol. This is in turn is converted to 25-hydroxyvitamin D (25-OHD) in the liver, which is further modified in the kidney by 1- $\alpha$ hydroxylase to $1,25(\mathrm{OH}) 2(2)$. In cells $1,25(\mathrm{OH}) 2$ binds to a vitamin D receptor (VDR), which then is able to migrate intracellularly, creating an intracellular signaling cascade, activing various cytokines with downstream modulating effects. The early literature focused on vitamin $\mathrm{D}$ and its receptor and the association with calcium, phosphorous, and bone metabolism. However, we 
have learned on a molecular level that VDR expression occurs throughout various cell lines, and in fact, is ubiquitous in cells that comprise our immune system, including macrophages, and T-cells. In macrophages, vitamin D is known to promote monocyte differentiation, antigen presentation, and the production of lysosomal enzyme activity, which are all critical in maintaining cellular hemostasis, and are impaired in the setting of its deficiency (1). Vitamin D deficiency has been shown to interfere with toll like receptor expression in dendrites, which deregulates cytokine production.

Vitamin D promotes synthesis of the anti-inflammatory cytokine Interleukin 10 (IL-10) and modulates tumor necrosis factor- $\alpha(\mathrm{TNF}-\alpha$,$) which is a key player in the$ pathogenicity of IBD. Briefly, IL-10 is an important antiinflammatory cytokine secreted by various cells, including monocytes, macrophages, and epithelial cells, and inhibits the release of TNF- $\alpha$. TNF- $\alpha$ is a known pro-inflammatory cytokine first recognized in 1969, where it was found to be a cytotoxic factor in lymphocytes based on its ability to kill mouse sarcoma cells. It is a pro-inflammatory cytokine which causes IL-10 dependent inhibition of CD 4 T-cell expansion, and promotes an inflammatory response in disorders including rheumatoid arthritis, ankylosing spondylitis, psoriasis, asthma, and IBD. Thus, IL-10 is critical for maintenance of immune homeostasis in the gastrointestinal tract (3). Further corroborating the role of IL-10, there are 40 case reports of individuals with very early onset IBD who have been found to be either IL-10 or IL-10 receptor deficient, with the only treatment being stem cell transplantation. Murine models also support this, showing IL-10 knockout mice develop spontaneous enterocolitis with severe diarrhea, which improves with vitamin D treatment (4). Furthermore, mice with an IL10 deficiency and knockout of their VDR develop an IBD phenotype with severe colitis which could not be rectified with vitamin $\mathrm{D}$, alluding to the role of vitamin $\mathrm{D}$ to potentiate the action of IL-10 and theoretically serve as a potential rescue therapy.

Vitamin D also exerts its potent affects via aiding in the formation of killer T-cells and CD8 T-cells, via cytokines, which have been shown to be important regulators of IBD (5). Our GI tract has a very unique population of CD8 T-Cells, which maintains tolerance to a large number of microbial food antigens in the gut. Effective CD8 T-cell function can suppress Th-1 and Th-17 helper T-cell activation, which leads to increased production of IL-17, a pro-inflammatory cytokine, and interferon gamma (IFN- $\gamma$ ), which increases intestinal permeability and worsens mucosal inflammation. In a synergistic way, vitamin D deficiency in mouse models can cause a 50-fold increase in colonic bacteria with increased translocation, postulated to be the result of the loss of VDR-dependent intercellular tight junctions (6). This provides two mechanisms through which vitamin $\mathrm{D}$ could affect mucosal integrity and cause injury.

As crucial as vitamin $\mathrm{D}$ is in affecting our immune system, VDR expression and functionality is also equally, if not more so, critical to its functionality. Abreu-Delgado et al. performed a case-control study in Puerto Rican patients with documented $\mathrm{UC}$ or $\mathrm{CD}$ and evaluated colonic biopsies using immunohistochemistry for VDR expression, as well as grade and severity of histological colitis on a scale of $1-3$. They found that patients with decreased colonic VDR expression had higher histological evidence of inflammation. There was a positive correlation between vitamin D levels and VDR expression in normal colonic mucosa, and negative correlation between VDR expression and inflammation in diseased mucosa (7). This is echoed in mouse models, showing mice genetically engineered to overexpress VDR in colonic mucosa actually have a decreased risk of colitis (8). Furthermore, we know that many intracellular pathogens play havoc by manipulating the immune system, and in fact, many do so by affecting VDR expression and thus the host immune response, including Mycobacterium Tuberculosis, EBV, HIV, which all have been shown to decrease VDR expression (9).

From an epidemiological perspective, geographical studies have revealed a north-south gradient for IBD, in which patients who live in northern regions, where sunlight exposure is decreased, have a higher incidence of IBD and also correlatively lower vitamin D levels. The European Collaborative Study on Inflammatory Bowel Disease reported an $80 \%$ greater incidence of Crohn's disease (CD) and $40 \%$ of ulcerative colitis (UC) when comparing patients from northern and southern European centers (1). An even larger retrospective study of over 72,000 women showed similar north-south latitude influence on incidence of IBD (10). It appears that low ultraviolet exposure was also associated with increased risk of hospitalization, prolonged length of stay, and IBD-related surgery, for both UC and $\mathrm{CD}$ (11). However, because vitamin $\mathrm{D}$ is not only generated by UVB exposure, but also can be considered a marker of exposure, it is often difficult to avoiding confounding in interpreting these studies.

Adding to confounding, there have been recent studies 
showing that in the setting of inflammation, vitamin $\mathrm{D}$ levels can be misleading, and decreased, in the acute phase across a wide array of illnesses. A large study by Ghashut et al. at the University of Glasgow looked to further explore the effect that systemic inflammation has on vitamin D levels. Between 2000 and 2013, vitamin D, CRP, and albumin levels were measured in acutely ill patients in the ICU with evidence of SIRS for a variety of diseases. All patients had elevated APACHE II scores, which is a validated score to predict ICU mortality. The study demonstrated that plasma concentrations of vitamin $\mathrm{D}$ correlated with changes in CRP and albumin. More specifically, the effect of CRP was most strongly associated with lower vitamin $\mathrm{D}$ levels, even when albumin was in the normal range $(\mathrm{P}<0.001)(12)$. Although mechanistically we are unclear about the pathogenesis, there is some postulated evidence that inflammatory cells, particularly macrophages, may have a consumptive effect on vitamin D in inflamed cells. In addition, the lower level may reflect transient consumption of body stores in a chronic ongoing systemic response. A study by Waldron et al. evaluated the vitamin D levels of thirty patients undergoing hip and knee surgery both pre- and post-surgical intervention, in addition to serum albumin and CRP. On average, vitamin D levels reduced by $40 \%$ on Day 2 post surgery, which also corresponded to the peak of the CRP levels that occurred on the same day. Following surgery, serum CRP increased in all patients and vitamin D levels decreased $(\mathrm{P}=0.0006)$ (13). Unfortunately, patients were not followed for a long enough period post-operatively to see if resolution of CRP also resulted in normalization of vitamin $\mathrm{D}$ to pre-surgical levels. Considering we often obtain vitamin D levels for our patients in the hospital when they are admitted for flares or at initial diagnosis when presenting in their most inflamed state, it is worth considering that perhaps our vitamin D levels are also falsely decreased. Therefore, we must also remain skeptical that vitamin $\mathrm{D}$ is an unreliable biomarker after an inflammatory insult, and consideration must be made on when it is obtained.

Unfortunately, there have overall been very few clinical studies to assess the role of vitamin D supplementation in IBD patients, and none in moderate to severe IBD (13). In a small, open-label study, vitamin D supplementation for six months improved quality of life scores in Crohn's patients with mild to moderate disease, as compared to placebo, stratifying across disease severity by the standardized Crohn's Disease Activity Index score, which is a validated clinical tool to assess severity of disease (5). A prospective study of oral vitamin D supplementation of 1,200 IU in adult patients in remission reduced the risk of relapse from $29 \%$ to $13 \%(\mathrm{P}=0.06)$ over the following year as compared to placebo. Although this was not statistically significant, the difference appeared to be clinically relevant (1). Perhaps the largest and most longitudinal study was performed at The University of Pittsburgh over a 5-year time frame. A total of 965 patients with the new diagnosis of IBD had vitamin D levels measured systematically and were supplemented, using a standardized protocol, with a low level being defined as less than $30 \mathrm{ng} / \mathrm{mL}$. During the analysis vitamin D levels were examined periodically, and patients were supplemented to try to achieve normal levels. Outcomes looked at the number of medications a patient was prescribed, number of IBD related emergency department visits, hospitalizations, surgeries, and quality of life. In the cohort, one-third had low vitamin levels to begin with and two thirds of those patients subsequently regained normal levels through supplementation interventions. Not surprisingly, it was shown that patients with low vitamin D levels at any given follow up time had a greater number of prescriptions for steroids, greater need for biological agents, higher mean disease activity scores, and a lower quality of life. Although the study did determine the association between vitamin $\mathrm{D}$ and various outcomes, alluding to the fact that normalization made a clinical improvement, it did not address the key question of causation (14).

Further studies looking into the role of supplementation in the context of CD therapy remain scarce. Krista Reich et al., at the University of Alberta, performed a prospective randomized control trial in patients with $\mathrm{CD}$ who were being induced with infliximab and stratified them into having low and normal vitamin D levels (15). These patients were followed prospectively for 14 weeks for achievement of clinical remission, and at week 14, vitamin $\mathrm{D}$ deficient patients were supplemented with intramuscular cholecalciferol and reassessed at week 22. Surprisingly, patients who had low vitamin D levels at onset had a higher rate of clinical remission compared to those with normal vitamin D Levels at both week 14 (80\% versus $23 \%$, $\mathrm{P}=0.007)$ and at week $22(79 \%$ versus $17 \%, \mathrm{P}=0.005$.) In fact, patients who were initiated on infliximab with low vitamin D levels were 3.5 times more likely to achieve remission. Two reasons have been given as a possible explanation. It is speculated that patients with low vitamin D levels may have a type of inflammatory response which 
would be most effectively treated with an anti-TNF- $\alpha$ such as infliximab. However, the study also assessed levels of TNF- $\alpha$ serologically and found that they were the same in both high and low vitamin D cohorts, making this theory unlikely. Given that vitamin D decreases proliferation of certain pro-inflammatory $\mathrm{T}$ lymphocytes, it is hypothesized that there may be more $\mathrm{T}$-cell proliferation in patients with $\mathrm{CD}$ with low vitamin $\mathrm{D}$ levels, and providing more cells for infliximab may be more effective in causing cellular apoptosis. Regardless, this study does illustrate that there is still much we do not understand about vitamin D mechanistically in IBD, and the surprising results certainly leave more questions than answers.

Causation $v s$. correlation unfortunately still remains the overwhelming issue in the elusive chicken vs egg debate. In essence it is understandable that if one has a more severe disease phenotype of IBD, he/she would have decreased absorption of vitamin $\mathrm{D}$ at the mucosal level secondary to malabsorption and therefore have lower levels detected serologically. Furthermore, if patients are significantly ill, then they are less likely to be outdoors, and hence would have decreased synthesis of cholecalciferol. On a cellular level, patients with IBD, particularly CD, also overexpress an enzyme in their colonic tissue known as CYP27B1, a cytochrome enzyme needed for the synthesis of $1,25(\mathrm{OH}) 2$. It is thought that this could be a protective mechanism to compensate for decreased vitamin $\mathrm{D}$ production in severe IBD to maintain localized levels in the colonic lumen (2).

In conclusion, Crohn's and UC are not diseases of vitamin $\mathrm{D}$ deficiency per se, but they clearly are diseases whose pathogenesis appears to be intimately linked to vitamin D at both the cellular and phenotypic level. Given the complexity of vitamin D and IBD, it is uncertain if causation will ever be equivocally established. However, we have strong support at least theoretically, and empirically from studies, that vitamin D plays a significant role in improving the disease state in IBD patients. Although we leave with more questions than answers, it is important to recognize that vitamin $\mathrm{D}$ is a player in the pathogenesis of IBD, and for now, our interventions should focus on obtaining vitamin D levels during asymptomatic periods, when possible, so as to avoid confounding, and investigating its potential synergy with current and future therapies in $\mathrm{CD}$ and IBD.

\section{Acknowledgements}

None.

\section{Footnote}

Conflicts of Interest: The authors have no conflicts of interest to declare.

\section{References}

1. Limketkai BN, Bechtold ML, Nguyen DL. Vitamin D and the Pathogenesis of Inflammatory Bowel Disease. Curr Gastroenterol Rep 2016;18:52.

2. Palmer MT, Weaver CT. Linking vitamin d deficiency to inflammatory bowel disease. Inflamm Bowel Dis 2013;19:2245-56.

3. Zhu L, Shi T, Zhong C, et al. IL-10 and IL-10 Receptor Mutations in Very Early Onset Inflammatory Bowel Disease. Gastroenterology Res 2017;10:65-9.

4. Cantorna MT, Munsick C, Bemiss C, et al. 1,25-Dihydroxycholecalciferol prevents and ameliorates symptoms of experimental murine inflammatory bowel disease. J Nutr 2000;130:2648-52.

5. Bora S, Cantorna MT. The role of UVR and vitamin D on T cells and inflammatory bowel disease. Photochem Photobiol Sci 2017;16:347-53.

6. Salim SY, Söderholm JD. Importance of disrupted intestinal barrier in inflammatory bowel diseases. Inflamm Bowel Dis 2011;17:362-81.

7. Abreu-Delgado Y, Isidro RA, Torres EA, et al. Serum vitamin $\mathrm{D}$ and colonic vitamin $\mathrm{D}$ receptor in inflammatory bowel disease. World J Gastroenterol 2016;22:3581-91.

8. Palmer MT, Lee YK, Maynard CL, et al. Lineage-specific effects of 1,25-dihydroxyvitamin $\mathrm{D}$ (3) on the development of effector CD4 T cells. J Biol Chem 2011;286:997-1004.

9. Mangin M, Sinha R, Fincher K. Inflammation and vitamin D: the infection connection. Inflamm Res 2014;63:803-19.

10. Khalili H, Huang ES, Ananthakrishnan AN, et al. Geographical variation and incidence of inflammatory bowel disease among US women. Gut 2012;61:1686-92.

11. Limketkai BN, Bayless TM, Brant SR, et al. Lower regional and temporal ultraviolet exposure is associated with increased rates and severity of inflammatory bowel disease hospitalisation. Aliment Pharmacol Ther 2014;40:508-17.

12. Ghashut RA, Talwar D, Kinsella J, et al. The effect of the systemic inflammatory response on plasma vitamin 25 $(\mathrm{OH}) \mathrm{D}$ concentrations adjusted for albumin. PLoS One 2014;9:e92614.

13. Waldron JL, Ashby HL, Cornes MP, et al. Vitamin D: a negative acute phase reactant. J Clin Pathol 
2013;66:620-2.

14. Ananthakrishnan AN. Editorial: Vitamin D and IBD: Can We Get Over the "Causation" Hump? Am J Gastroenterol 2016;111:720-2.

doi: $10.21037 / \operatorname{tgh} .2018 .05 .03$

Cite this article as: Mudambi K, Bass D. Vitamin D: a brief overview of its importance and role in inflammatory bowel disease. Transl Gastroenterol Hepatol 2018;3:31.
15. Reich KM, Fedorak RN, Madsen K, et al. Role of Vitamin $\mathrm{D}$ in Infliximab-induced Remission in Adult Patients with Crohn's Disease. Inflamm Bowel Dis 2016;22:92-9. 